PROCEEDINGS OF THE

AMERICAN MATHEMATICAL SOCIETY

Volume 131, Number 11, Pages 3371-3378

S 0002-9939(03)06930-2

Article electronically published on February 24, 2003

\title{
FULLY COMMUTATIVE ELEMENTS AND KAZHDAN-LUSZTIG CELLS IN THE FINITE AND AFFINE COXETER GROUPS
}

\author{
JIAN-YI SHI \\ (Communicated by John R. Stembridge)
}

\begin{abstract}
The main goal of the paper is to show that the fully commutative elements in the affine Coxeter group $\widetilde{C}_{n}$ form a union of two-sided cells. Then we completely answer the question of when the fully commutative elements of $W$ form or do not form a union of two-sided cells in the case where $W$ is either a finite or an affine Coxeter group.
\end{abstract}

Let $W$ be a Coxeter group with $S$ the distinguished generator set. The fully commutative elements of $W$ were defined by Stembridge: $w \in W$ is fully commutative if any two reduced expressions of $w$ can be transformed from each other by only applying the relations $s t=t s$ with $s, t \in S$ and $o(s t)=2$, or equivalently, $w$ has no reduced expression of the form $w=x(s t s \ldots) y$, where $s t s \ldots$ is a string of length $o(s t)>2(o(s t)$ being the order of $s t)$ for some $s \neq t$ in $S$. The fully commutative elements were studied extensively by a number of people (see [3], [6], [8], [16]). Now let $W$ be either a finite or an affine Coxeter group and let $W_{\mathrm{c}}$ be the set of all the fully commutative elements in $W$. We consider the relation between $W_{\text {c }}$ and the two-sided cells of $W$ (in the sense of Kazhdan and Lusztig, see [9]). It is well known that when $W$ is either the finite Coxeter group $A_{n}(n \geqslant 1), B_{l}$ $(l \geqslant 2), I_{2}(m)(m \geqslant 2)$, or the affine Coxeter group $\widetilde{A}_{n}(n \geqslant 1), W_{\mathrm{c}}$ is a union of two-sided cells of $W$ (see [12, §1.7, Theorems 16.2.8 and 17.4], [13, Theorem 3.1] and [8, Theorem 3.1.1]). On the other hand, since $W_{\mathrm{c}}$ is not a union of two-sided cells of $W$ when $W=D_{4}$ (see [2]), it should also be the case when $W$ contains a standard parabolic subgroup of type $D_{4}$, i.e., $W$ is $D_{n}, \widetilde{D}_{n}, \widetilde{B}_{n}, E_{m}, \widetilde{E}_{m}(n \geqslant 4$, $m=6,7,8)$. Recently, R. M. Green asked the following question (see [7, §4]): is $W_{\text {c }}$ a union of two-sided cells of $W$ for $W=\widetilde{C}_{n}$ ? The present paper will give an affirmative answer to the question (Theorem 3.4). Furthermore, we completely answer the question of when $W_{\mathrm{c}}$ is or is not a union of two-sided cells of $W$ in the case where $W$ is either a finite or an affine Coxeter group.

The contents of the paper are organized as follows. Section 1 contains preliminaries; some definitions and results are collected there. Then we show some properties of the elements in $\widetilde{C}_{n}$ preserved under star operations in Section 2. Finally, we prove our main result in Section 3.

Received by the editors May 1, 2002 and, in revised form, May 28, 2002 and June 13, 2002.

2000 Mathematics Subject Classification. Primary 20F55, 05E15.

The author was partially supported by Nankai University, the 973 Project of MST of China, the NSF of China, the SF of the University Doctorial Program of ME of China and the Shanghai Priority Academic Discipline. 


\section{$\S 1$. Preliminaries}

1.1. The affine Coxeter group $\widetilde{C}_{n}$ can be identified with the following permutation group over the integer set $\mathbb{Z}$ (see [13 $\$ 1.4]$ ):

$\widetilde{C}_{n}=\{\sigma: \mathbb{Z} \longrightarrow \mathbb{Z} \mid(i+2 n+2) \sigma=(i) \sigma+2 n+2$ and $(-i) \sigma=-(i) \sigma$ for $i \in \mathbb{Z}\}$,

where we assume $n \geqslant 2$ throughout the paper. Its Coxeter generator set $S=\left\{s_{i} \mid\right.$ $0 \leqslant i \leqslant n\}$ is given as follows. For $k \in \mathbb{Z}$ and $1 \leqslant i<n$, we have

$$
\begin{gathered}
(k) s_{i}= \begin{cases}k, & \text { if } k \not \equiv \pm i, \pm(i+1)(\bmod 2 n+2), \\
k+1, & \text { if } k \equiv i,-i-1(\bmod 2 n+2), \\
k-1, & \text { if } k \equiv i+1,-i(\bmod 2 n+2),\end{cases} \\
(k) s_{0}= \begin{cases}k, & \text { if } k \neq \equiv \pm(\bmod 2 n+2), \\
k+2, & \text { if } k \equiv-1(\bmod 2 n+2), \\
k-2, & \text { if } k \equiv 1(\bmod 2 n+2),\end{cases} \\
(k) s_{n}= \begin{cases}k, & \text { if } k \neq n, n+2(\bmod 2 n+2), \\
k+2, & \text { if } k \equiv n(\bmod 2 n+2), \\
k-2, & \text { if } k \equiv n+2(\bmod 2 n+2) .\end{cases}
\end{gathered}
$$

1.2. In $1.2-1.4$, let $W$ be a Coxeter group with $S$ the distinguished generator set. Let $\ell(w)$ be the length function of $W$ and $\leqslant$ the Bruhat order on $W$ with respect to $S$. For any $w \in W$, let $\mathcal{L}(w)=\{s \in S \mid s w<w\}$ and $\mathcal{R}(w)=\{s \in S \mid w s<w\}$. When $W=\widetilde{C}_{n}$ and $0 \leqslant i \leqslant n$, we have $s_{i} \in \mathcal{L}(w)$ if and only if $(i) w>(i+1) w$ (see [13, Proposition 1.6]). In particular, this implies that $s_{0} \in \mathcal{L}(w)$ if and only if $(-1) w>(1) w$ and that $s_{n} \in \mathcal{L}(w)$ if and only if $(n) w>(n+2) w$. The set $\mathcal{R}(w)$ can be described similarly by using the fact $\mathcal{R}(w)=\mathcal{L}\left(w^{-1}\right)$.

1.3. Following [10, for any $s, t \in S$ with the order $m=o(s t)$ of the product st greater than 2 , we call any of the sequences sy,tsy, stsy, .. and ty, sty,tsty,... (each has $m-1$ terms) an $\{s, t\}$-string if $\mathcal{L}(y) \cap\{s, t\}=\emptyset$. When $w$ is a term of some $\{s, t\}$-string, a transformation sending $w$ to one of its neighboring terms in the string is called an $\{s, t\}$-star operation (or a star operation in short). Note that a star operation defined here is slightly different from that by Kazhdan and Lusztig in [9]; the latter was defined only in the case of $m=3$. For any $w \in W$, let $M(w)$ be the set of all the elements $y$ such that there exists a sequence of elements $z_{0}=w, z_{1}, \ldots, z_{t}=y$ in $W$ with $t \geqslant 0$ such that $z_{i}$ is obtained from $z_{i-1}$ by a star operation for every $1 \leqslant i \leqslant t$. The sequence $z_{0}, z_{1}, \ldots, z_{t}$ is called a path in $M(w)$ from $w$ to $y$ (or a path in $M(w)$ ). Two elements $x, y \in W$ have the same generalized $\tau$-invariants if for any path $z_{0}=x^{-1}, z_{1}, \ldots, z_{t}$ in $M\left(x^{-1}\right)$ there is a path $z_{0}^{\prime}=y^{-1}, z_{1}^{\prime}, \ldots, z_{t}^{\prime}$ in $M\left(y^{-1}\right)$ with $\mathcal{L}\left(z_{i}^{\prime}\right)=\mathcal{L}\left(z_{i}\right)$ for every $0 \leq i \leq t$, and if the same condition holds when interchanging the roles of $x$ with $y$.

1.4. The preorders $\underset{L}{\leq}, \underset{R}{\leq}, \underset{L R}{\leq}$ and the associated equivalence relations $\underset{L}{\sim}, \underset{R}{\sim}, \underset{L R}{\sim}$ on $(W, S)$ are defined as in [9]. The equivalence classes of $W$ with respect to $\widetilde{L}($ resp., $\underset{R}{\sim}$, resp., $\underset{L R}{\widetilde{N}}$ ) are called left cells (resp., right cells, resp., two-sided cells). It follows easily from the definition of a left cell that $x \underset{L}{\sim} y$ for any $w \in W$ 
and any $x, y \in M(w)$. It is well known that if $x, y \in W$ satisfy $x \underset{L}{\sim} y$, then $x, y$ have the same generalized $\tau$-invariants (see [14, Proposition 4.2]).

1.5. For $w \in \widetilde{C}_{n}$, call an integer sequence $\xi: i_{1}, i_{2}, \ldots, i_{r}$ a $w$-chain if

(1) $i_{1}<i_{2}<\ldots<i_{r}$ and $\left(i_{1}\right) w>\left(i_{2}\right) w>\ldots>\left(i_{r}\right) w$

(2) either $(2 \mathrm{a}) i_{j}+i_{k} \not \equiv 0(\bmod 2 n+2)$ for any $1 \leqslant j<k \leqslant r$, or $(2 \mathrm{~b}) r$ is even and $i_{j}+i_{r+1-j} \equiv 0(\bmod 2 n+2)$ for $1 \leqslant j \leqslant r$;

(3) $i_{h} \not \equiv 0, n+1(\bmod 2 n+2)$ for $1 \leqslant h \leqslant r$.

Note that condition (1) implies that $i_{1}, i_{2}, \ldots, i_{r}$ are pairwise noncongruent modulo $2 n+2$.

A $w$-chain $\xi$ as above is of type I (resp., II) if it satisfies (2a) (resp., (2b)). Define the length of $\xi$ to be $r$ (resp., $\frac{r}{2}+1$ ) if $\xi$ is of type I (resp., II). Comparing with the terminology in [13, a $w$-chain of type II is just a union of chains in a special chain pair of $w$ defined in [13, $\S 2.4]$, and a $w$-chain of type $\mathrm{I}$ is a chain of $w$ in [13], but not all the chains of $w$ in [13] are of type $\mathrm{I}$.

1.6. For a $w$-chain $\xi: i_{1}, i_{2}, \ldots, i_{p}$ and $q \in \mathbb{Z}$, the sequences $\xi_{q}: q(2 n+2)+i_{1}$, $q(2 n+2)+i_{2}, \ldots, q(2 n+2)+i_{r}$ and $\xi_{q}^{\prime}: q(2 n+2)-i_{r}, q(2 n+2)-i_{r-1}, \ldots, q(2 n+2)-i_{1}$ with some $q \in \mathbb{Z}$ are also $w$-chains. Call $\xi_{q}$ a chain-shifting of $\xi$, call $\xi_{q}^{\prime}$ a chainreflection of $\xi$, and call both chain-replacements of $\xi$.

\section{§2. SOME PROPERTIES PRESERVED BY STAR OPERATIONS}

In the present section, we show some properties of the elements in $\widetilde{C}_{n}$ which are preserved by star operations (see Lemmas 2.2 and 2.3). The property in Lemma 2.3 is crucial in the proof of Theorem 3.4.

For $w, x, y \in \widetilde{C}_{n}$, we use the notation $w=x \cdot y$ to mean $w=x y$ and $\ell(w)=$ $\ell(x)+\ell(y)$. According to [13, Theorem 3.2], we have the following:

Lemma 2.1. Let $w \in \widetilde{C}_{n}$. Then $w$ is not fully commutative if and only if there exists a $w$-chain of length $\geqslant 3$. More precisely, $w=x \cdot s_{i} s_{i+1} s_{i} \cdot y$ for some $x, y \in \widetilde{C}_{n}$ and $1 \leqslant i<n-1$ if and only if there exists a w-chain of type $I$ and length $\geqslant 3$. Also, $w=x \cdot s_{k} s_{k+1} s_{k} s_{k+1} \cdot y$ for some $x, y \in \widetilde{C}_{n}$ and $k \in\{0, n-1\}$ if and only if there exists a w-chain of type $I I$ and length $\geqslant 3$.

Since the terminology used here and in 13 is different, we have to use the present terminology to illustrate how [13, Theorem 3.2] implies Lemma 2.1. In [13, §2.4], we defined a chain pair $P, P^{\prime}$ of $w$, where $P$ is a $w$-chain of type $I$ and $P^{\prime}=\{2 n+2-x \mid x \in P\}$ such that $P \cap P^{\prime}=\emptyset$. In [13, §2.5], we also defined $d^{\prime}(w)_{1}$ as the maximal possible value for $\left|P \cup P^{\prime}\right|+\epsilon(w, P)$, where $P, P^{\prime}$ range over chain pairs of $w$, and $\epsilon(w, P)$ is 1 or 0 according to whether $P$ could be or could not be a part of some $w$-chain of type II. Let $J$ be a proper subset of $\{0,1, \ldots, n\}$ consisting of consecutive integers and let $w_{J}$ be the longest element in the subgroup of $\widetilde{C}_{n}$ generated by $s_{i}, i \in J$. In [13, (2.9.3)], we defined the value $d_{1}^{\prime}\left(w_{J}\right)$ which is $2|J|+2$ if $0, n \notin J$ and $2|J|+1$ (correction: the number $2|J|+1$ was misprinted as $2(|J|+1)+1$ in [13. (2.9.2)]) if otherwise. Then [13, Theorem 3.2] tells us that there exists an expression $w=x \cdot w_{J} \cdot y$ for some $x, y \in \widetilde{C}_{n}$ and some consecutive integer subset $J$ of $\{0,1, \ldots, n\}$ with $d_{1}^{\prime}\left(w_{J}\right)=d_{1}^{\prime}(w)$ and that there does not exist any expression of the form $w=x^{\prime} \cdot w_{I} \cdot y^{\prime}$ for any $x^{\prime}, y^{\prime} \in \widetilde{C}_{n}$ and any consecutive integer subset $I$ of $\{0,1, \ldots, n\}$ with $d_{1}^{\prime}\left(w_{I}\right)>d_{1}^{\prime}(w)$. This implies that $w$ is not 
fully commutative if and only if $d_{1}^{\prime}(w) \geqslant 5$, and the latter holds if and only if there exists a $w$-chain of length $\geqslant 3$.

Now we consider some properties of elements in $\widetilde{C}_{n}$ preserved by star operations.

Lemma 2.2. Assume that $y, w \in \widetilde{C}_{n}$ can be obtained from each other by an $\left\{s_{i}, s_{i+1}\right\}$-star operation for some $1 \leqslant i<n-1$. If there exists a w-chain $\xi$, then there exists a $y$-chain of the same type and length as $\xi$.

Proof. Let $\xi: i_{1}, i_{2}, \ldots, i_{r}$. Then $y=s_{t} w$ for some $t \in\{i, i+1\}$. If either $\ell(y)=$ $\ell(w)+1$ or

$(*)(t, t+1) \notin\left\{\left(q(2 n+2)+i_{h}, q(2 n+2)+i_{h+1}\right),\left(q(2 n+2)-i_{h+1}, q(2 n+2)-i_{h}\right)\right\}$

for any $1 \leqslant h<r$ and $q \in \mathbb{Z}$, then $\left(i_{1}\right) s_{t},\left(i_{2}\right) s_{t}, \ldots,\left(i_{r}\right) s_{t}$ is a $y$-chain of the same type as the $w$-chain $\xi$. Now assume that we are not in any of the above cases, that is, we assume that $\ell(y)=\ell(w)-1$ and that

$$
(t, t+1) \in\left\{\left(q(2 n+2)+i_{h}, q(2 n+2)+i_{h+1}\right),\left(q(2 n+2)-i_{h+1}, q(2 n+2)-i_{h}\right)\right\}
$$

for some $1 \leqslant h<r$ and $q \in \mathbb{Z}$. The result will be shown by finding some $w$ chain $\xi^{\prime}$ of the same type and length as $\xi$ and satisfying condition $(*)$ with $\xi^{\prime}$ in the place of $\xi$. Applying a suitable chain-replacement on $\xi$ if necessary, we have $\left(i_{h}, i_{h+1}\right)=(t, t+1)$ for some $1 \leqslant h<r$. Then either $(t) w>(t-1) w>(t+1) w$ or $(t) w>(t+2) w>(t+1) w$ holds by the assumption that $s_{t} w$ can be obtained from $w$ by a star operation. Clearly, for any $1 \leqslant j \leqslant r$, we have $i_{j} \not \equiv t-1(\bmod 2 n+2)$ in the former case, and $i_{j} \not \equiv t+2(\bmod 2 n+2)$ in the latter case. We only deal with the former case and then the latter case can be done similarly, so we assume we are in the former case. First assume that $i_{k} \neq q(2 n+2)-(t-1)$ for any $1 \leqslant k \leqslant r$ and $q \in \mathbb{Z}$. When $\xi$ is of type I, we replace $i_{h}$ by $t-1$ in $\xi$. When $\xi$ is of type II, we replace $i_{h}, i_{r+1-h}$ by $i_{h}-1, i_{r+1-h}+1$, respectively, in $\xi$. Next assume that $i_{k}=q(2 n+2)-(t-1)$ for some $1 \leqslant k \leqslant r$ and $q \in \mathbb{Z}$ (hence $\xi$ is of type I). When $q>0$, i.e., $k>h+1$, we replace $\xi$ by $q(2 n+2)-i_{r}, q(2 n+2)-$ $i_{r-1}, \ldots, q(2 n+2)-i_{k}, i_{h+1}, i_{h+2}, \ldots, i_{k-1}, q(2 n+2)-i_{h}, \ldots, q(2 n+2)-i_{1}$. When $q \leqslant 0$, i.e., $k<h$, we replace $\xi$ by $i_{1}, \ldots, i_{k-1}, q(2 n+2)-i_{h}, q(2 n+2)-i_{h-1}, \ldots$, $q(2 n+2)-i_{k}, i_{h+1}, i_{h+2}, \ldots, i_{r}$. In either case, we get a new $w$-chain, say $\xi^{\prime}$, which is of the same type and length as $\xi$. Also, $\xi^{\prime}$ satisfies condition $(*)$ with $\xi^{\prime}$ in the place of $\xi$. This proves our result.

The conclusion of Lemma 2.2 no longer holds in general if $w, y \in \widetilde{C}_{n}$ can be obtained from each other either by an $\left\{s_{0}, s_{1}\right\}$-star operation or by an $\left\{s_{n-1}, s_{n}\right\}$ star operation. However, we have the following:

Lemma 2.3. Suppose that $y, w \in \widetilde{C}_{n}$ can be obtained from each other by an $\left\{s_{i}, s_{i+1}\right\}$-star operation with $0 \leqslant i \leqslant n-1$. If there exists a w-chain of length $\geqslant 3$, then there also exists a $y$-chain of length $\geqslant 3$.

Proof. When $1 \leqslant i<n-1$, the result follows from Lemma 2.2. Thus it remains to consider the case $i=0, n-1$. We shall only deal with the case $i=n-1$. Then the case $i=0$ can be discussed similarly. Now assume $i=n-1$. Let $\xi: i_{1}, i_{2}, \ldots, i_{r}$ be a $w$-chain with $r \geqslant 3$. Assume that $y=s_{t} w$ with $t \in\{n-1, n\}$. We may assume that $\left(i_{1}\right) s_{t},\left(i_{2}\right) s_{t}, \ldots,\left(i_{r}\right) s_{t}$ is not a $y$-chain (hence $\left.\ell(y)=\ell(w)-1\right)$ since otherwise we are done. Then what we want to do is to find a $w$-chain $\xi^{\prime}: j_{1}, j_{2}, \ldots, j_{u}$ with $u \geqslant 3$ such that $\left(j_{1}\right) s_{t},\left(j_{2}\right) s_{t}, \ldots,\left(j_{u}\right) s_{t}$ is a $y$-chain. Applying a suitable chain-replacement if 
necessary, we may assume that either $t=n-1,\left(i_{h}, i_{h+1}\right)=(n-1, n)$ or $t=n$, $\left(i_{h}, i_{h+1}\right)=(n, n+2)$ holds.

(1) First assume $t=n-1$ and $\left(i_{h}, i_{h+1}\right)=(n-1, n)$. We claim that $\xi$ is of type I; otherwise, $\xi$ is of type II. Since $n-1, n$ are two terms of $\xi, \xi$ should also contain two terms $q(2 n+2)-n, q(2 n+2)-n+1$ for some $q \in \mathbb{Z}$. If $q>0$, then $n-1, n, n+2, n+3$ would form a $w$-chain. If $q \leqslant 0$, then we would have $(n) w<$ $(n-1) w<(n+3) w<(n+2) w$. None of these cases could happen since $s_{n-1} w$ can be obtained from $w$ by an $\left\{s_{n-1}, s_{n}\right\}$-star operation. This proves the claim. We have either $(n+3) w<(n) w<(n+2) w<(n-1) w$ or $(n) w<(n+3) w<(n-1) w<$ $(n+2) w$. In the former case, $n$ is replaced by $n+2$ in $\xi$. In the latter case, if $h>1$, then $\xi$ is replaced by $\xi^{\prime}: i_{1}, \ldots, i_{h-1}, n-1, n+3,2 n+2-i_{h-1}, \ldots, 2 n+2-i_{1}$; if $h=1$, then $\xi$ is replaced by $\xi^{\prime}: 2 n+2-i_{r}, 2 n+2-i_{r-1}, \ldots, 2 n+2-i_{3}, n-1, n+3, i_{3}, \ldots, i_{r}$.

(2) Next assume $t=n$ and $\left(i_{h}, i_{h+1}\right)=(n, n+2)$. Then $\xi$ has type II and $r=2 h$ is even with $h>1$. We have either $(n+2) w<(n+3) w<(n-1) w<(n) w$ or $(n+2) w<(n-1) w<(n+3) w<(n) w$. In either case, $\xi$ is replaced by $\xi^{\prime}: i_{1}, i_{2}, \ldots, i_{h-1}, n-1, n+2$.

Clearly, in any of the above cases, we get a required $w$-chain $\xi^{\prime}$. This proves our result.

Remark 2.4. By Lemmas 2.3 and 2.1, we see that the property of being fully commutative (or equivalently, not being fully commutative) is preserved under star operations on the elements in $\widetilde{C}_{n}$.

\section{$\S 3$. Main RESUlts}

Let $W$ be a finite or an affine Coxeter group. In this section, we shall answer the question of when the set of the fully commutative elements of $W$ is or is not a union of two-sided cells. The main part of the section is concerned with the case $W=\widetilde{C}_{n}$.

Call $J \subset S$ fully commutative if $s t=t s$ for any $s, t \in J$.

Lemma 3.1. Let $w \in \widetilde{C}_{n}$ be fully commutative with $J=\mathcal{L}(w)$. If $\mathcal{L}(s w) \subset \mathcal{L}(w)$ for any $s \in J$, then $w$ is fully commutative.

Proof. By 1.2 and the assumption on $w$, we have

(i) $(i-1) w<(i+1) w<(i) w<(i+2) w$ if $s_{i} \in J$ and $1 \leqslant i<n$;

(ii) $(-2) w<(1) w<0<(-1) w<(2) w$ if $s_{0} \in J$;

(iii) $(n-1) w<(n+2) w<n+1<(n) w<(n+3) w$ if $s_{n} \in J$.

By 1.2 , we also have

(iv) $(k) w<(k+1) w$ if $s_{k} \notin J$ and $1 \leqslant k<n,(-1) w<0<(1) w$ if $s_{0} \notin J$, and $(n) w<n+1<(n+2) w$ if $s_{n} \notin J$.

Suppose that $J=\left\{s_{i_{j}} \mid 1 \leqslant j \leqslant r, i_{1}<i_{2}<\ldots<i_{r}\right\}$. Then we see from (i)-(iv) that

(v) $\left(i_{1}-\delta_{i_{1}, 0}\right) w<\left(i_{2}\right) w<\ldots<\left(i_{r}\right) w$ and $\left(i_{1}+1\right) w<\left(i_{2}+1\right) w<\ldots<$ $\left(i_{r}+1+\delta_{i_{r}, n}\right) w$, where $\delta_{h j}=0$ or 1 according to whether $h \neq j$ or $h=j$;

(vi) $(h) w<(j) w<(k) w$ for any $-1 \leqslant h<j<k \leqslant n+2$ with $j \notin\left\{i_{m}, i_{m}+1 \mid\right.$ $1 \leqslant m \leqslant r\}$.

Let $I_{i}=S \backslash\left\{s_{i}\right\}$ and $J_{j}=I_{j} \backslash\left\{s_{j+1}\right\}$ (set difference) for $0 \leqslant i \leqslant n$ and $0 \leqslant j<n$.

Let $W_{I}$ be the subgroup of $\widetilde{C}_{n}$ generated by $I$ for $I \subseteq S$. Then we see that

(vii) if $s_{0} \notin J$, then $0<(h) w<2 n+2$ for $0<h<2 n+2$ and so $w \in W_{I_{0}}$; 
(viii) if $s_{n} \notin J$, then $-n-1<(h) w<n+1$ for $-n-1<h<n+1$ and so $w \in W_{I_{n}}$

(ix) if $\left\{s_{j}, s_{j+1}\right\} \cap J=\emptyset$ with some $1 \leqslant j<n-1$, then $-(j+1) w<(h) w<$ $(j+1) w$ for $-j-1<h<j+1$ and $(j+1) w<(k) w<(2 n+1-j) w$ for $j+1<k<2 n+1-j$. This implies that $(j+1) w=j+1$ and hence $w \in W_{J_{j}}$.

In any of the cases (vii)-(ix), any $w$-chain can be chain-replaced into the closed interval $[a, a+2 n]$ for some integer $a$. More precisely, we can take $a=1$ (resp., $a=-n$, resp., $a=-j$ ) in the case (vii) (resp., (viii), resp., (ix)). Then it is easily seen from (v)-(vi) that there is no $w$-chain of length $\geqslant 3$ in any of the cases (vii)-(ix).

(x) If $n$ is even and $J=\left\{s_{0}, s_{2}, s_{4}, \ldots, s_{n}\right\}$, then (1) $w<(3) w<\ldots<$ $(n-1) w<(n+2) w<(n+4) w<\ldots<(2 n) w<(2 n+3) w=2 n+2+(1) w$ and $(-1) w<(2) w<(4) w<\ldots<(n) w<(n+3) w<(n+5) w<\ldots<(2 n+1) w=$ $2 n+2+(-1) w$. Let $\mathbb{Z}_{2 n+2}$ be the set of residue classes of $\mathbb{Z}$ modulo $2 n+2$. Then $\mathbb{Z}_{2 n+2}$ is a disjoint union of two subsets

$$
E_{1}=\{\overline{1}, \overline{3}, \ldots, \overline{n-1}, \overline{n+2}, \overline{n+4}, \ldots, \overline{2 n}\}
$$

and

$$
E_{2}=\{\overline{-1}, \overline{2}, \overline{4}, \ldots, \bar{n}, \overline{n+3}, \overline{n+5}, \ldots, \overline{2 n-1}\}
$$

For any $w$-chain $\xi: i_{1}, i_{2}, \ldots, i_{r}$, denote by $\bar{\xi}$ the set $\left\{\overline{i_{j}} \mid 1 \leqslant j \leqslant r\right\}$. Then we see that the set $\bar{\xi} \cap E_{k}$ contains at most one element for any $k=1,2$. This implies that there is no $w$-chain of length $\geqslant 3$ in this case.

Since at least one of the cases (vii)-(x) must occur by the fully commutativity of $J$, we conclude that $w$ is fully commutative by Lemma 2.1 .

Lemma 3.2. Let $w \in \widetilde{C}_{n}$ be not fully commutative. Then there exists some element $y \in M(w)$ such that $\mathcal{L}(y)$ is not fully commutative.

Proof. The result is obvious when either $n>2, \ell(w)=3$, or $n=2, \ell(w)=4$, since $y=w$ must be a required element in $M(w)$. Now assume $\ell(w)>3$. If $\mathcal{L}(w)$ is not fully commutative, then $y=w$ is a required element in $M(w)$. Now assume that $\mathcal{L}(w)$ is fully commutative. By Lemma 3.1, there exists some $s \in \mathcal{L}(w)$ with $\mathcal{L}(s w) \nsubseteq \mathcal{L}(w)$. Hence $w^{\prime}=s w$ is in $M(w)$. By Lemma 2.1, there exists a $w$-chain of length $\geqslant 3$. Then by Lemma 2.3 , there exists a $w^{\prime}$-chain of length $\geqslant 3$. Our result follows by applying induction on $\ell(w) \geqslant 3$ when $n>2$, or on $\ell(w) \geqslant 4$ when $n=2$.

Corollary 3.3. An element $w \in \widetilde{C}_{n}$ is not fully commutative if and only if there exists some $y \in M(w)$ such that $\mathcal{L}(y)$ is not fully commutative.

Proof. The implication " $\Longrightarrow$ " follows by Lemma 3.2. Then the reverse implication follows by Remark 2.4 .

Let $W_{\mathrm{c}}$ be the set of all the fully commutative elements in $\widetilde{C}_{n}$ and let $W_{\mathrm{c}}^{\prime}$ be the complementary set of $W_{\text {c }}$ in $\widetilde{C}_{n}$.

Theorem 3.4. The set $W_{\mathrm{c}}$ is a union of two-sided cells of $\widetilde{C}_{n}$.

Proof. Suppose not. Then there must exist some $x \in W_{\mathrm{c}}$ and $y \in W_{\mathrm{c}}^{\prime}$ satisfying $x \underset{L R}{\sim} y$. We know that the intersection of the left cell containing $x$ and the right cell containing $y$ is nonempty (it follows easily by the associativity of the Hecke 
algebra of $W$ and by [11] Corollary 1.9]). So we may take some $z$ with $x \underset{L}{\sim} \underset{R}{\sim} y$. We see that an element $x \in \widetilde{C}_{n}$ is fully commutative if and only if so is the element $x^{-1}$. Thus there is no loss in assuming $x \underset{L}{\sim} y$ to begin with. By Corollary 3.3, we see that there exists some $y^{\prime} \in M\left(y^{-1}\right)$ with $\mathcal{L}\left(y^{\prime}\right)$ not fully commutative and that $\mathcal{L}\left(x^{\prime}\right)$ is fully commutative for any $x^{\prime} \in M\left(x^{-1}\right)$. This contradicts the fact that $x, y$ have the same generalized $\tau$-invariants (see 1.4). Our result follows.

3.5. By the knowledge of their two-sided cells, we see that an element $w$ of $W$ is not fully commutative if and only if there exists some $y \in M(w)$ such that $\mathcal{L}(y)$ is not fully commutative in the case where $W$ is one of the following groups: $A_{n}, \widetilde{A}_{n}$ $\left(n \geqslant 1\right.$; see [12] Propositions 16.2.4 and 9.3.7] and [13, Theorem 3.1]), $B_{m}(m \geqslant 2$; see [8, Theorem 3.1.1]), $F_{4}$ (see [17]), $I_{2}(m)\left(m \geqslant 2\right.$; see [12, §1.7]), $H_{3}$ and $H_{4}$ (see [1]), $\widetilde{G}_{2}$ (see [10]). In this case, we conclude that the fully commutative elements of $W$ do form a union of two-sided cells.

3.6. Let $W$ be a finite or affine Coxeter group with a branching Coxeter graph, i.e., $W$ is one of the following groups: $D_{n}, \widetilde{D}_{n}(n \geqslant 4), \widetilde{B}_{l}(l \geqslant 3), E_{m}, \widetilde{E}_{m}(m=6,7,8)$. Then the fully commutative elements do not form a union of two-sided cells. This is because the group $W$ either contains a standard parabolic subgroup $D_{4}$ or is $\widetilde{B}_{3}$ and because the fully commutative elements in any of the groups $D_{4}$ and $\widetilde{B}_{3}$ do not form a union of two-sided cells. Let $\left\{s_{1}, s_{2}, s_{3}, s_{4}\right\}$ be the Coxeter generator set of the group $D_{4}$ with $o\left(s_{1} s_{2}\right)=o\left(s_{2} s_{3}\right)=o\left(s_{2} s_{4}\right)=3$. Then $s_{1} s_{3} s_{4} \underset{L R}{\sim} s_{1} s_{2} s_{1}$, where $s_{1} s_{3} s_{4}$ is fully commutative, but $s_{1} s_{2} s_{1}$ is not (see [2]). Let $S=\left\{s_{0}, s_{1}, s_{2}, s_{3}\right\}$ be the Coxeter generator set of the group $\widetilde{B}_{3}$ with $o\left(s_{0} s_{2}\right)=o\left(s_{1} s_{2}\right)=3$ and $o\left(s_{2} s_{3}\right)=4$. Then $s_{0} s_{1} s_{3} \underset{L R}{\sim} s_{0} s_{2} s_{0}$, where $s_{0} s_{1} s_{3}$ is fully commutative but $s_{0} s_{2} s_{0}$ is not (see 4]).

3.7. In the affine Coxeter group $\widetilde{F}_{4}$, the fully commutative elements do not form a union of two-sided cells. Let $\left\{s_{0}, s_{1}, s_{2}, s_{3}, s_{4}\right\}$ be a Coxeter generator set of $\widetilde{F}_{4}$ with $o\left(s_{0} s_{1}\right)=o\left(s_{1} s_{2}\right)=o\left(s_{3} s_{4}\right)=3$ and $o\left(s_{2} s_{3}\right)=4$. Then $s_{0} s_{1} s_{0} \underset{L R}{\sim} s_{0} s_{2} s_{4}$ (see [15, §5.4]). Clearly, $s_{0} s_{2} s_{4}$ is fully commutative, but $s_{0} s_{1} s_{0}$ is not.

\section{ACKNOWLEDGEMENT}

I am very grateful to the referee for his/her invaluable comments on this paper. I would like to take this opportunity to express my deep gratitute to Professor Y. C. Chen who invited me to visit Nankai University and to the Center for Combinatorics, Nankai University, for their hospitality during the writing of this paper.

\section{REFERENCES}

1. D. Alvis and G. Lusztig, The representations and generic degrees of the Hecke algebra of type $H_{4}$, J. Reine Angew. Math. 336 (1982), 201-212. MR 84a:20013

2. D. Barbasch and D. Vogan, Primitive ideals and Orbital integrals in complex classical groups, Math. Ann. 259 (1982), 153-199. MR 83m:22026

3. S. C. Billey and G. S. Warrington, Kazhdan-Lusztig polynomials for 321-hexagon-avoiding permutations, J. Algebraic Combin. 13 (2001), 111-136. MR 2002f:05161

4. Jie $\mathrm{Du}$, The decomposition into cells of the affine Weyl group of type $\widetilde{B}_{3}$, Comm. Algebra 16 (1988), 1383-1409. MR 89i:20074 
5. C. K. Fan, A Hecke algebra quotient and properties of commutative elements of a Weyl group, Ph.D. thesis, M.I.T., 1995.

6. C. K. Fan and J. R. Stembridge, Nilpotent orbits and commutative elements, J. Algebra 196 (1997), 490-498. MR 98g:20067

7. R. M. Green, On 321-avoiding permutations in affine Weyl groups, J. Algebraic Combin. 15 (2002), 241-252.

8. R. M. Green and J. Losonczy, Fully commutative Kazhdan-Lusztig cells, Ann. Inst. Fourier (Grenoble) 51 (2001), 1025-1045. MR 2002e:20076

9. D. Kazhdan and G. Lusztig, Representations of Coxeter groups and Hecke algebras, Invent. Math. 53 (1979), 165-184. MR 81j:20066

10. G. Lusztig, Cells in affine Weyl groups, in "Algebraic Groups and Related Topics"(R. Hotta, ed.), Advanced Studies in Pure Math., Kinokuniya and North Holland, (1985), 255-287. MR 87h:20074

11. G. Lusztig, Cells in affine Weyl groups, II, J. Algebra 109 (1987), 536-548. MR 88m:20103a

12. Jian-yi Shi, The Kazhdan-Lusztig cells in certain affine Weyl groups, vol. 1179, SpringerVerlag, Lecture Notes in Math., 1986. MR 87i:20074

13. Jian-yi Shi, Some results relating two presentations of certain affine Weyl groups, J. Algebra 163(1) (1994), 235-257. MR 94k:20076

14. Jian-yi Shi, Left cells in affine Weyl groups, Tohoku J. Math. 46 (1994), 105-124. MR 94k:20077

15. Jian-yi Shi, Left cells in the affine Weyl group of type $\widetilde{F}_{4}$, J. Algebra 200 (1998), 173-206. MR 99b:20069

16. J. R. Stembridge, On the fully commutative elements of Coxeter groups, J. Algebraic Combin. 5 (1996), 353-385. MR 97g:20046

17. K. Takahashi, The left cells and their $W$-graphs of Weyl group of type $F_{4}$, Tokyo J. Math. 13 (1990), 327-340. MR 92a:20048

Center for Combinatorics, The Key laboratory of Pure Mathematics and Combinatorics of Ministry of Education, Nankai University, Tianjin, 300071, People's Republic of China - And - Department of Mathematics, East China Normal University, Shanghai, 200062, People's Republic of China

E-mail address: jyshi@math.ecnu.edu.cn 